\title{
The Low Energy Effective Lagrangian for Photon Interactions in Any Dimension
}

\author{
A. Ritz and R. Delbourgo \\ Department of Physics, University of Tasmania \\ GPO Box 252C Hobart, Tasmania, Australia 7001.
}

\begin{abstract}
The subject of low energy photon-photon scattering is considered in arbitrary dimensional space-time and the interaction is widened to include scattering events involving an arbitrary number of photons. The effective interaction Lagrangian for these processes in QED has been determined in a manifestly invariant form. This generalisation resolves the structure of the weak-field Euler-Heisenberg Lagrangian and indicates that the component invariant functions have coefficients related, not only to the space-time dimension, but also to the coefficients of the Bernoulli polynomial.
\end{abstract}

\section{Introduction}

That an interaction between electromagnetic fields and the vacuum of the Dirac field would allow such nonlinear processes as the scattering of photons by photons has been known for some time. Even before the emergence of the full scale theory of quantum electrodynamics (QED) Halpern, Euler, and Heisenberg, among others, discussed the phenomenon in the mid 1930s [1, 2, 3, ㄴ. ‥ However, it was in the early nineteen fifties that the subject was considered in detail using QED, and Karplus \& Neuman [5, 6] determined the full photon-photon scattering cross-section.

The work presented in this paper is related to a desire to generalise the discussion of photon interactions to arbitrary dimensional space-time and to consider how the parameters of the interaction change in this case, and also with the number of scattering photons. This will be done by investigating the underlying effective Lagrangian for the interaction, and little consideration will be given to measureable cross-sections. The reason for this being that the cross-section determined by Karplus \& Neuman, and others subsequently [7], for four photon scattering in four dimensional space-time is extremely small (i.e. $\sigma \sim 10^{-30} \mathrm{~cm}^{2}$ ). This is perhaps not unexpected for such an interaction, but it does mean that obtaining experimentally testable values is of limited importance at this stage. The generalisation to arbitrary dimensional space-time 
serves a twofold purpose. Firstly, it is useful to know the form of the result in the context of dimensional regularisation. More importantly perhaps it will provide greater insight into the structure of the Lagrangian in the dimensions of practical interest, i.e. $D=2,3$ and 4 . As a scattering process involving the fewest number of photons (i.e. four) will have the largest cross-section, generalisation to interactions involving an arbitrary number of photons will also primarily be of interest in further resolving the structure in the Lagrangian.

A scattering process involving $N$ photons can be represented by a sum over permutations of an $N$-photon electron loop. i.e. a coupling of $N$ photons via an intermediate loop of electron and positron propagators. Direct evaluation of the Feynman amplitude in this case would be quite involved and this problem is most easily tackled by taking, as a starting point, the full 1-loop Lagrangian for the field involving a summation of all possible couplings between the electromagnetic field and a virtual matter field. For reasons of clarity it is useful to split the discussion of $N$-photons interactions into two cases: those involving an even number of photons; and those involving an odd number. We shall proceed now to consider the first of these.

\section{$2 \quad 2 \mathrm{~N}-\mathrm{Photon}$ Case}

Interactions involving an even number of photons particularly lend themselves to the term photon-photon scattering, as a situation involving the same number of initial and final photons can easily be envisaged. In the discussion which follows we shall implicitly assume that the interactions involve $2 N$ photons, where $N$ is integral, and thus the number of interaction photons involved is always even. In order to discuss these cases we can recall the 1-loop electromagnetic field Lagrangians obtained by Brown \& Duff [8] for the cases of bosonic and fermionic virtual particle loops:

$$
\begin{aligned}
& \mathcal{L}_{I}^{(0)}= \frac{1}{(4 \pi)^{D / 2}} \int_{0}^{\infty} \frac{\mathrm{d} s}{s^{1+D / 2}} e^{-m^{2} s} \exp \left[-\frac{1}{2} \operatorname{Tr} \ln \left(\frac{\sin e F s}{e F s}\right)\right], \\
& \mathcal{L}_{I}^{(1 / 2)}=-\frac{1}{2(4 \pi)^{D / 2}} \int_{0}^{\infty} \frac{\mathrm{d} s}{s^{1+D / 2}} e^{-m^{2} s} \operatorname{Tr} \exp \left[\frac{1}{2} e s \sigma \cdot F\right] \\
& \exp \left[-\frac{1}{2} \operatorname{Tr} \ln \left(\frac{\sin e F s}{e F s}\right)\right] .
\end{aligned}
$$

These results being generalisations of the four-dimensional Lagrangians obtained by Schwinger [9, 10]. Brown \& Duff used momentum space functional methods to consider the Lagrangian in any field theory due to 1-loop quantum vacuum effects. The reader is directed to their paper for details of the derivation, while we shall simply use their results to consider the case of $2 N$-photon scattering. It should be noted that these expressions are valid in strong fields as they contain all orders of the coupling to the electromagnetic field. Taking only the lowest order terms will correspond to the situation of weak fields.

As it is a simple starting point we shall first consider the situation of photon scattering via a virtual meson loop (i.e. spin zero propagator particles). Techniques 
used in this case will then be applied to the slightly more complex process in which photon scattering is mediated by an electron/positron loop.

\subsection{Scalar Case}

Our starting point here will be the 1-loop Lagrangian of Brown \& Duff (Eq. 目) which can be written in the following form

$$
\mathcal{L}_{I}^{(0)}=\frac{1}{(4 \pi)^{D / 2}} \int_{0}^{\infty} \frac{\mathrm{d} s}{s^{1+D / 2}} e^{-m^{2} s} \exp \left[\frac{1}{2} \operatorname{Tr} \ln f(i F)\right]
$$

where

$$
f(z)=\frac{e s z}{\sinh (e s z)}
$$

This Lagrangian, although it must be Lorentz covariant, is not explicitly so. Our task will be to extract a given order of the interaction (i.e. $2 N$-photon scattering) and express this part of the Lagrangian in a manifestly covariant form. To this end we can follow the procedure described by Delbourgo \& Matsuki [11] and suppose that the function $f(z)$ possesses a series expansion of the form

$$
f(z)=1+\sum_{n=1}^{\infty} b_{n} x^{2 n}
$$

which is clearly true in the case above. Then writing the exponential and logarithmic functions as power series we can obtain

$$
\mathcal{L}_{I}^{(0)}=\frac{1}{(4 \pi)^{D / 2}} \int_{0}^{\infty} \frac{\mathrm{d} s}{s^{1+D / 2}} e^{-m^{2} s} \sum_{l=0}^{\infty} \frac{1}{l !}\left[\operatorname{Tr} \sum_{m=1}^{\infty} a_{m} F^{2 m}\right]^{l},
$$

where new constants $a_{m}$ have been introduced to simplify the expression. As this relation is now in the form of a single power series expansion we can pick out any given term, and in particular the general term, which corresponds to a $2 N$-photon scattering interaction, will be given by

$$
\begin{aligned}
\mathcal{L}_{I}^{(0)}= & \frac{1}{(4 \pi)^{D / 2}} \int_{0}^{\infty} \frac{\mathrm{d} s}{s^{1+D / 2}} e^{-m^{2} s} \sum_{\left\{n_{i}\right\}}^{n_{1}+2 n_{2}+\cdots+N n_{N}=N} \frac{1}{n_{1} ! n_{2} ! \ldots n_{N} !} \\
& \times\left(a_{1} \operatorname{Tr} F^{2}\right)^{n_{1}}\left(a_{2} \operatorname{Tr} F^{4}\right)^{n_{2}} \cdots\left(a_{N} \operatorname{Tr} F^{2 N}\right)^{n_{N}} .
\end{aligned}
$$

In this expression the summation over $n_{i}$ runs from zero to a certain integer such that the partitioning constraint,

$$
n_{1}+2 n_{2}+3 n_{3}+\cdots+N n_{N}=N
$$

is satisfied. We should note here that implicit in this selection of a particular order of the expansion as a valid Lagrangian is any renormalisation of charge, and corresponding scale changes for the fields, which may be needed. 
This Lagrangian is now in a manifestly invariant form and the problem has reduced to determining the coefficients $a_{m}$. Fortunately a method for determining these factors has been developed in a rather different area of field theory. Delbourgo \& Matsuki [11], when considering gravitational and chiral anomalies [12, 13] obtained the following expression in terms of a two-form matrix version of the Riemann tensor $R$

$$
A_{D}=C_{D} \exp \left[\frac{1}{2} \operatorname{Tr} \ln f(i R)\right] .
$$

As we see this is identical to the second exponential term in the 1-loop Lagrangian (Eq. 3) apart from the use of $R$ in place of $F$. The fact that the same mathematics has turned up in apparently unrelated areas of field theory is certainly of note, but leaving this interesting point aside we can use the following expression, obtained by Delbourgo \& Matsuki [11], to determine the coefficients $a_{m}$.

$$
a_{m}=\frac{i(-1)^{m+1}}{8 \pi m} \oint \mathrm{d} z z^{-2 m} \frac{\mathrm{d}}{\mathrm{d} z} \ln f(z) .
$$

This general result can be applied to the particular case of the spin-0 Lagrangian in which we have

$$
f(z)=\frac{e s z}{\sinh (e s z)}
$$

Determination of the coefficients can then be achieved by performing the integration and noting that

$$
\frac{z}{e^{z}-1}=\sum_{n=0}^{\infty} \frac{B_{n}}{n !} z^{n},
$$

where $B_{n}$ are the Bernoulli numbers. We obtain

$$
a_{m}=\frac{(-1)^{m+1}(2 e s)^{2 m}}{4 m} \frac{B_{2 m}}{(2 m) !} .
$$

Thus, for a $2 N$-photon process mediated by a virtual meson loop, the interaction Lagrangian is given by

$$
\begin{aligned}
\mathcal{L}_{I}^{(0)}= & \frac{1}{(4 \pi)^{D / 2}} \int_{0}^{\infty} \frac{\mathrm{d} s}{s^{1+D / 2}} e^{-m^{2} s} e^{2 N} s^{2 N} \sum_{\left\{n_{i}\right\}}^{n_{1}+2 n_{2}+\cdots+N n_{N}=N} \frac{1}{n_{1} ! n_{2} ! \ldots n_{N} !} \\
& \times\left(a_{1}^{\prime} \operatorname{Tr} F^{2}\right)^{n_{1}}\left(a_{2}^{\prime} \operatorname{Tr} F^{4}\right)^{n_{2}} \cdots\left(a_{N}^{\prime} \operatorname{Tr} F^{2 N}\right)^{n_{N}}
\end{aligned}
$$

where

$$
a_{m}^{\prime}=\frac{(-1)^{m+1} 2^{2 m}}{4 m} \frac{B_{2 m}}{(2 m) !} .
$$

We can now proceed to perform the integration, obtaining

$$
\begin{aligned}
\mathcal{L}_{I}^{(0)}= & \frac{1}{(4 \pi)^{D / 2}} \frac{e^{2 N}}{\left(m^{2}\right)^{2 N-D / 2}} \int_{0}^{\infty}\left(m^{2} s\right)^{(2 N-D / 2)-1} e^{-m^{2} s} \mathrm{~d}\left(m^{2} s\right) \\
& \sum_{\left\{n_{i}\right\}}^{n_{1}+2 n_{2}+\cdots+N n_{N}=N} \frac{1}{n_{1} ! n_{2} ! \ldots n_{N} !}\left(a_{1}^{\prime} \operatorname{Tr} F^{2}\right)^{n_{1}}\left(a_{2}^{\prime} \operatorname{Tr} F^{4}\right)^{n_{2}} \cdots\left(a_{N}^{\prime} \operatorname{Tr} F^{2 N}\right)^{n_{N}} .
\end{aligned}
$$


The integral in this expression is simply the defining relation for the $\Gamma$-function, i.e.

$$
\Gamma(x)=\int_{0}^{\infty} t^{x-1} e^{-t} \mathrm{~d} t,
$$

and thus

$$
\begin{aligned}
\mathcal{L}_{I}^{(0)}= & \frac{\left(e^{2}\right)^{N}}{(4 \pi)^{D / 2}} \frac{1}{\left(m^{2}\right)^{2 N-D / 2}} \Gamma(2 N-D / 2) \sum_{\left\{n_{i}\right\}}^{n_{1}+2 n_{2}+\cdots+N n_{N}=N} \frac{1}{n_{1} ! n_{2} ! \ldots n_{N} !} \\
& \times\left(a_{1}^{\prime} \operatorname{Tr} F^{2}\right)^{n_{1}}\left(a_{2}^{\prime} \operatorname{Tr} F^{4}\right)^{n_{2}} \cdots\left(a_{N}^{\prime} \operatorname{Tr} F^{2 N}\right)^{n_{N}} .
\end{aligned}
$$

We will find that it is particularly illuminating in the spinor case to use the the coefficients of the Bernoulli polynomial $B_{n}(x)$ [14],

$$
\frac{z e^{x z}}{e^{z}-1}=\sum_{n=0}^{\infty} B_{n}(x) \frac{z^{n}}{n !},
$$

in place of the Bernoulli numbers and for later comparison we shall introduce them here. With the substitution $B_{n}(0)=B_{n}$ the invariant coefficients become

$$
a_{m}^{\prime}=\frac{(-1)^{m+1} 2^{2 m}}{4 m(2 m) !} B_{2 m}(0),
$$

and we can write the effective interaction Lagrangian in the following compact form

$$
\begin{aligned}
\mathcal{L}_{I}^{(0)}= & \frac{\left(e^{2}\right)^{N}}{(4 \pi)^{D / 2}} \frac{1}{\left(m^{2}\right)^{2 N-D / 2}} \Gamma(2 N-D / 2) \sum_{\left\{n_{i}\right\}}^{n_{1}+2 n_{2}+\cdots+N n_{N}=N} \frac{1}{n_{1} ! n_{2} ! \ldots n_{N} !} \\
\times\left(\frac{B_{2}(0)}{2} \operatorname{Tr} F^{2}\right)^{n_{1}}\left(-\frac{B_{4}(0)}{12} \operatorname{Tr} F^{4}\right)^{n_{2}} & \ldots\left(\frac{(-1)^{N+1} 2^{2 N}}{4 N(2 N) !} B_{2 N}(0) \operatorname{Tr} F^{2 N}\right)^{n_{N}} .
\end{aligned}
$$

This is our final expression for the Lorentz invariant low energy effective interaction Lagrangian for the electromagnetic field due to spin-0 quantum loop effects. Although this superficially describes all $2 N$-photon scattering interactions in $D$ dimensions, we should note that in some cases the $\Gamma$-function may be undefined, i.e. for large even $D$ and small $N$. This is not a serious problem as in the cases of most interest (e.g. 4 photon scattering in $D=4$ ) the result will be either finite or manageable via regularisation.

As a particular example we can consider the case of four photon scattering $(N=2)$ in four dimensional space-time. In this case the constraint over the $\left\{n_{i}\right\}$ implies that the only two terms allowed will have $n_{1}=2$ and $n_{2}=1$. Thus we immediately recover the electromagnetic field invariants

$$
\left(\operatorname{Tr} F^{2}\right)^{2}, \quad \text { and } \quad \operatorname{Tr} F^{4},
$$

obtained in previous discussions of four photon scattering. The coefficients can be evaluated easily from the general expression, and in this case we obtain

$$
\mathcal{L}_{I}^{(0)}=\frac{\alpha^{2}}{90 m^{4}}\left[\frac{5}{16}\left(\operatorname{Tr} F^{2}\right)^{2}+\frac{1}{4} \operatorname{Tr} F^{4}\right],
$$

which is precisely the low energy limit of the result obtained by Schwinger [9, 10]. 


\subsection{Spinor Case}

For this case our starting point is the spin-1/2 1-loop Lagrangian of Brown \& Duff (Eq. 2) where we see the spinor case introduces the extra trace over spinor indices,

$$
\operatorname{Tr} \exp \left[\frac{1}{2} e s \sigma \cdot F\right] .
$$

Before proceeding to use the techniques developed in the scalar case this trace must be evaluated and in order to do so it is neccessary to consider the cases of odd and even dimensional space-times separately.

\subsubsection{Even $D$}

Evaluation of the spinor trace is essentially a purely mathematical exercise, and in order to simplify the process as much as possible we can perform the calculation in Euclidean rather than Minkowskian space. In this case the metric, $g_{\mu \nu}$, assumes the form of the identity matrix and for even dimensions the antisymmetric electromagnetic field tensor can be written in the following canonical form,

$$
(F)^{\mu \nu}=\left(\begin{array}{ccccccc}
0 & \lambda_{1} & 0 & & \cdots & & \\
-\lambda_{1} & 0 & & & & & \\
0 & & 0 & \lambda_{2} & & & \\
& & -\lambda_{2} & 0 & & & \\
\vdots & & & & \ddots & & \\
& & & & & 0 & \lambda_{D / 2} \\
& & & & & -\lambda_{D / 2} & 0
\end{array}\right),
$$

where $\lambda_{i}$ are the eigenvalues. This canonical form can be achieved by a similarity transformation, which will not effect the commutation relations between the Lorentz group generators, but corresponds to choosing some canonical basis for the $\gamma$-matrices.

If we consider a coupling of the electromagnetic field to a spin- $1 / 2$ field via

$$
\sigma_{\mu \nu}=\frac{i}{2}\left[\gamma_{\mu}, \gamma_{\nu}\right]=\frac{i}{2}\left(\gamma_{\mu} \gamma_{\nu}-\gamma_{\nu} \gamma_{\mu}\right)
$$

then this will result in the invariant matrix $\sigma \cdot F$ which we can represent as

$$
\frac{1}{2} \sigma F=\frac{1}{2} i \gamma_{\mu} \gamma_{\nu} F^{\mu \nu}=i\left(\gamma_{0} \gamma_{1} F^{01}+\cdots+\gamma_{D-2} \gamma_{D-1} F^{D-2 D-1}\right)
$$

Noting that $\left(\gamma_{\mu}\right)^{2}=\mathbf{1}$, i.e the identity matrix, the defining anticommutation relations imply

$$
\left(\gamma_{i} \gamma_{i+1}\right)^{2}=\mathbf{1}, \quad \forall i=0 . .(D-2) .
$$

Consequently we can perform an expansion of the following exponential

$$
\begin{aligned}
\exp \left(i \gamma_{0} \gamma_{1} F^{01}\right) & =\mathbf{1}+i \gamma_{0} \gamma_{1} F^{01}-\frac{\left(F^{01}\right)^{2}}{2 !} \mathbf{1}-i \gamma_{0} \gamma_{1} \frac{\left(F^{01}\right)^{3}}{3 !}+\cdots \\
& =\cos F^{01} \mathbf{1}+i \gamma_{0} \gamma_{1} \sin F^{01}
\end{aligned}
$$


which, on consideration of the canonical representation for the tensor $F^{\mu \nu}$, gives

$$
\begin{aligned}
\exp \left(i \gamma_{0} \gamma_{1} F^{01}\right) & =\cos \lambda_{1} \mathbf{1}+i \gamma_{0} \gamma_{1} \sin \lambda_{1} \\
& =\left(\mathbf{1}-i \gamma_{0} \gamma_{1} \frac{\mathrm{d}}{\mathrm{d} \lambda_{1}}\right) \cos \lambda_{1}
\end{aligned}
$$

This result allows the exponential function of the full invariant matrix $\sigma \cdot F$,

$$
\exp \left(\frac{1}{2} \sigma F\right)=\exp \left(i \gamma_{0} \gamma_{1} F^{01}+\cdots+i \gamma_{D-2} \gamma_{D-1} F^{D-2 D-1}\right),
$$

where incidentally all the matrices in the exponent commute with each other, to be evaluated as

$$
\begin{aligned}
& \exp \left(\frac{1}{2} \sigma F\right)=\exp \left(i \gamma_{0} \gamma_{1} F^{01}\right) \exp \left(i \gamma_{2} \gamma_{3} F^{23}\right) \cdots \exp \left(i \gamma_{D-2} \gamma_{D-1} F^{D-2 D-1}\right) \\
& =\left(\mathbf{1}-i \gamma_{0} \gamma_{1} \frac{\partial}{\partial \lambda_{1}}\right) \cdots\left(\mathbf{1}-i \gamma_{D-2} \gamma_{D-1} \frac{\partial}{\partial \lambda_{D / 2}}\right)\left[\cos \lambda_{1} \cdots \cos \lambda_{D / 2}\right] \\
& =\left[\begin{array}{rl}
1 & +\left(i \gamma_{0} \gamma_{1} \frac{\partial}{\partial \lambda_{1}}+i \gamma_{2} \gamma_{3} \frac{\partial}{\partial \lambda_{2}}+\cdots\right) \\
& -\left(i \gamma_{0} \gamma_{1} \gamma_{2} \gamma_{3} \frac{\partial}{\partial \lambda_{1} \partial \lambda_{2}}+\cdots\right) \\
& +\cdots \\
& -\left(\gamma_{1} \gamma_{2} \cdots \gamma_{D-2} \gamma_{D-1} \frac{\partial}{\partial \lambda_{1} \cdots \partial \lambda_{D / 2}}\right)
\end{array}\right]\left[\cos \lambda_{1} \cdots \cos \lambda_{D / 2}\right] .
\end{aligned}
$$

Hence if we take the trace of this expression we obtain

$$
\begin{aligned}
\operatorname{Tr} \exp \left(\frac{1}{2} \sigma F\right) & =\operatorname{Tr}(\mathbf{1})\left[\cos \lambda_{1} \cdots \cos \lambda_{D / 2}\right] \\
& =2^{[D / 2]} \prod_{j=1}^{D / 2} \cos \lambda_{j} \\
& =2^{[D / 2]} \exp \left[\frac{1}{2} \operatorname{Tr} \ln \cos F\right],
\end{aligned}
$$

where the factor of $1 / 2$ which enters in the last line is due to the form of the canonical representation for $F^{\mu \nu}$. Substituting this evaluated form of the spinor trace into the effective Lagrangian results in

$$
\mathcal{L}_{I}^{(1 / 2)}=-\frac{2^{[D / 2]}}{2(4 \pi)^{D / 2}} \int_{0}^{\infty} \frac{\mathrm{d} s}{s^{1+D / 2}} e^{-m^{2} s} \exp \left[\frac{1}{2} \operatorname{Tr} \ln f(i F)\right],
$$

where

$$
f(z)=\frac{e s z}{\tanh e s z} .
$$

This is precisely the form required to use the techniques developed in the scalar case. We will proceed with this process later on, although now we should consider evaluation of the spinor trace for odd dimensional space-times. 


\subsubsection{Odd $D$}

In Euclidean space the canonical representation for the electromagnetic field tensor is given by the even dimensional case augmented by a zero eigenvalue $\lambda_{0}=0$. This is a consequence of the form of the characteristic identity for an odd-dimensional antisymmetric matrix which implies the existence of at least one vanishing eigenvalue. If we label the eigenvalues $\lambda_{0}=0, \lambda_{1}, \lambda_{2}, \ldots, \lambda_{[D / 2]}$ then the analysis can proceed in an analogous manner to the even dimensional case. i.e. we obtain

$$
\operatorname{Tr} \exp \left(\frac{1}{2} \sigma F\right)=\operatorname{Tr}(\mathbf{1})\left[\cos 0 \cos \lambda_{1} \cdots \cos \lambda_{[D / 2]}\right],
$$

where the extra eigenvalue has been included for clarity. Our labelling of the eigenvalues now means that this expression is in exactly the same form as the even dimensional case and thus the interaction Lagrangian

$$
\mathcal{L}_{I}^{(1 / 2)}=-\frac{2^{[D / 2]}}{2(4 \pi)^{D / 2}} \int_{0}^{\infty} \frac{\mathrm{d} s}{s^{1+D / 2}} e^{-m^{2} s} \exp \left[\frac{1}{2} \operatorname{Tr} \ln f(i F)\right],
$$

where

$$
f(z)=\frac{e s z}{\tanh e s z}
$$

applies in a space-time of arbitrary dimension.

We may wonder at this stage as to the nature of any parity violating terms which may appear in odd dimensional space-times. These terms actually arise via the free field Lagrangian and must be considered separately, as we shall subsequently do.

\subsubsection{Evaluation of the Effective Interaction Lagrangian}

Having performed our manipulation of the Brown \& Duff spin-1/2 1-loop Lagrangian into the form given by Eq. 7 we can follow our discussion of the scalar case, extracting a given order of the interaction (i.e. $2 N$-photon scattering), and expressing it in the following manifestly covariant form

$$
\begin{aligned}
\mathcal{L}_{I}^{(1 / 2)}=- & \frac{2^{[D / 2]}}{2(4 \pi)^{D / 2}} \int_{0}^{\infty} \frac{\mathrm{d} s}{s^{1+D / 2}} e^{-m^{2} s} \sum_{\left\{n_{i}\right\}}^{n_{1}+2 n_{2}+\cdots+N n_{N}=N} \frac{1}{n_{1} ! n_{2} ! \ldots n_{N} !} \\
& \times\left(a_{1} \operatorname{Tr} F^{2}\right)^{n_{1}}\left(a_{2} \operatorname{Tr} F^{4}\right)^{n_{2}} \ldots\left(a_{N} \operatorname{Tr} F^{2 N}\right)^{n_{N}} .
\end{aligned}
$$

The technique of Delbourgo \& Matsuki [11] can now be used to determine the coefficients $a_{m}$ in an analogous manner to the calculation in the scalar case.

Following this procedure we have

$$
a_{m}=\frac{i(-1)^{m+1}}{8 \pi m} \oint \mathrm{d} z z^{-2 m} \frac{\mathrm{d}}{\mathrm{d} z} \ln f(z),
$$

where in the case of the spin-1/2 Lagrangian

$$
f(z)=\frac{e s z}{\tanh (e s z)}
$$


The integration in this case makes use of the following identities

$$
\begin{aligned}
& \frac{z}{e^{z}-1}=\sum_{n=0}^{\infty} \frac{B_{n}}{n !} z^{n} \\
& \frac{z}{e^{z}+1}=\sum_{n=0}^{\infty} \frac{B_{n}}{n !}\left(1-2^{n}\right) z^{n}
\end{aligned}
$$

where, again, the symbol $B_{n}$ represents the Bernoulli numbers. We obtain in this case

$$
a_{m}=\frac{(-1)^{m+1}(2 e s)^{2 m}}{4 m} \frac{B_{2 m}}{(2 m) !}\left(2-2^{2 m}\right) .
$$

Hence, for a $2 N$-photon process mediated by a virtual spin- $1 / 2$ fermion loop, the interaction Lagrangian is given by

$$
\begin{aligned}
\mathcal{L}_{I}^{(1 / 2)}=- & \frac{2^{[D / 2]}}{2(4 \pi)^{D / 2}} \int_{0}^{\infty} \frac{\mathrm{d} s}{s^{1+D / 2}} e^{-m^{2} s} e^{2 N} s^{2 N} \sum_{\left\{n_{i}\right\}}^{n_{1}+2 n_{2}+\cdots+N n_{N}=N} \frac{1}{n_{1} ! n_{2} ! \ldots n_{N} !} \\
& \times\left(a_{1}^{\prime} \operatorname{Tr} F^{2}\right)^{n_{1}}\left(a_{2}^{\prime} \operatorname{Tr} F^{4}\right)^{n_{2}} \cdots\left(a_{N}^{\prime} \operatorname{Tr} F^{2 N}\right)^{n_{N}}
\end{aligned}
$$

where

$$
a_{m}^{\prime}=\frac{(-1)^{m+1} 2^{2 m}}{4 m} \frac{B_{2 m}}{(2 m) !}\left(2-2^{2 m}\right) .
$$

The final integration can be performed as in the scalar case giving

$$
\begin{aligned}
\mathcal{L}_{I}^{(1 / 2)}= & -\frac{\left(e^{2}\right)^{N}}{(4 \pi)^{D / 2}} \frac{2^{[D / 2]-1}}{\left(m^{2}\right)^{2 N-D / 2}} \Gamma(2 N-D / 2) \sum_{\left\{n_{i}\right\}}^{n_{1}+2 n_{2}+\cdots+N n_{N}=N} \frac{1}{n_{1} ! n_{2} ! \ldots n_{N} !} \\
& \times\left(a_{1}^{\prime} \operatorname{Tr} F^{2}\right)^{n_{1}}\left(a_{2}^{\prime} \operatorname{Tr} F^{4}\right)^{n_{2}} \cdots\left(a_{N}^{\prime} \operatorname{Tr} F^{2 N}\right)^{n_{N}} .
\end{aligned}
$$

The expression comes of course with the proviso that the $\Gamma$-function is finite or can at least be handled via regularisation.

We can obtain a compact form for the effective Lagrangian by noting the following correspondance between the Bernoulli numbers and the coefficients of the Bernoulli polynomial [14],

$$
\left(2-2^{n}\right) B_{n}=2^{n} B_{n}\left(\frac{1}{2}\right)
$$

With this substitution the coefficients are given by

$$
a_{m}^{\prime}=\frac{(-1)^{m+1} 2^{4 m}}{4 m(2 m) !} B_{2 m}\left(\frac{1}{2}\right)
$$

and we can write the effective interaction Lagrangian in the following form

$$
\begin{aligned}
\mathcal{L}_{I}^{(1 / 2)}= & -\frac{\left(e^{2}\right)^{N}}{(4 \pi)^{D / 2}} \frac{2^{D / 2]-1}}{\left(m^{2}\right)^{2 N-D / 2}} \Gamma(2 N-D / 2) \sum_{\left\{n_{i}\right\}}^{n_{1}+2 n_{2}+\cdots+N n_{N}=N} \frac{1}{n_{1} ! n_{2} ! \ldots n_{N} !} \\
& \times\left(2 B_{2}\left(\frac{1}{2}\right) \operatorname{Tr} F^{2}\right)^{n_{1}}\left(-\frac{4}{3} B_{4}\left(\frac{1}{2}\right) \operatorname{Tr} F^{4}\right)^{n_{2}} \\
& \cdots\left(\frac{(-1)^{N+1} 2^{4 N}}{4 N(2 N) !} B_{2 N}\left(\frac{1}{2}\right) \operatorname{Tr} F^{2 N}\right)^{n_{N}} .
\end{aligned}
$$


Hence the full effective Lagrangian, obtained by adding in the free field Lagrangian, is given in almost all dimensions by

$$
\begin{aligned}
\mathcal{L}^{(1 / 2)}= & -\frac{1}{4} \operatorname{Tr} F^{2} \\
& -\frac{\left(e^{2}\right)^{N}}{(4 \pi)^{D / 2}} \frac{2^{D / 2]-1}}{\left(m^{2}\right)^{2 N-D / 2}} \Gamma(2 N-D / 2) \sum_{\left\{n_{i}\right\}}^{n_{1}+2 n_{2}+\cdots+N n_{N}=N} \frac{1}{n_{1} ! n_{2} ! \ldots n_{N} !} \\
& \times\left(2 B_{2}\left(\frac{1}{2}\right) \operatorname{Tr} F^{2}\right)^{n_{1}}\left(-\frac{4}{3} B_{4}\left(\frac{1}{2}\right) \operatorname{Tr} F^{4}\right)^{n_{2}} \\
& \cdots\left(\frac{(-1)^{N+1} 2^{4 N}}{4 N(2 N) !} B_{2 N}\left(\frac{1}{2}\right) \operatorname{Tr} F^{2 N}\right)^{n_{N}} .
\end{aligned}
$$

The qualification is placed on this statement due to the existence of certain parity violating terms present in odd dimensional space-times which are expected due to the presence of fermion mass terms in the classical action. Delbourgo \& Waites 15 have shown that these terms have the form

$$
C \epsilon_{\mu_{1} \mu_{2} \cdots \mu_{D}} A^{\mu_{1}} F^{\mu_{2} \mu_{3}} \cdots F^{\mu_{D-1} \mu_{D}} .
$$

Due to the order of this expression in the electromagnetic field tensor $F$, it is apparent that this term can only relate to a $2 N$-photon process such that

$$
D=2(2 N)-1
$$

The relationship implies that in only one dimension $D$, for a given $2 N$-photon interaction, will the parity violating term emerge. i.e. for a 2 photon process $D=3$, for a 4 photon process $D=7$, etc. Interestingly, these are the dimensions for which a charge conjugation operator exists. Delbourgo \& Waites have determined the general form of the coefficient $C$ which, for an interaction of $2 N$ soft photons, is given by

$$
C=\frac{e^{2 N}}{2(2 N) !(4 \pi)^{2 N-1}} .
$$

Hence, for such a process in a space-time of dimension $D=2(2 N)-1$ ( $N$ integral of course), the effective Lagrangian is given by

$$
\begin{aligned}
\mathcal{L}^{(1 / 2)}= & -\frac{1}{4} \operatorname{Tr} F^{2} \\
& -\frac{\left(e^{2}\right)^{N}}{(4 \pi)^{D / 2}} \frac{2^{D / 2]-1}}{\left(m^{2}\right)^{2 N-D / 2}} \Gamma(2 N-D / 2) \sum_{\left\{n_{i}\right\}}^{n_{1}+2 n_{2}+\cdots+N n_{N}=N} \frac{1}{n_{1} ! n_{2} ! \ldots n_{N} !} \\
& \times\left(2 B_{2}\left(\frac{1}{2}\right) \operatorname{Tr} F^{2}\right)^{n_{1}}\left(-\frac{4}{3} B_{4}\left(\frac{1}{2}\right) \operatorname{Tr} F^{4}\right)^{n_{2}} \\
& \ldots\left(\frac{(-1)^{N+1} 2^{4 N}}{4 N(2 N) !} B_{2 N}\left(\frac{1}{2}\right) \operatorname{Tr} F^{2 N}\right)^{n_{N}} \\
& +\frac{e^{2 N}}{2(2 N) !(4 \pi)^{2 N-1}} \epsilon_{\mu_{1} \mu_{2} \cdots \mu_{D}} A^{\mu_{1}} F^{\mu_{2} \mu_{3}} \ldots F^{\mu_{D-1} \mu_{D}} .
\end{aligned}
$$


In practice the only term of major importance is likely to be that of two photon vacuum polarisation in $D=3$, the extra invariant in this case being the Chern-Simons term. It is however useful, for reasons of generality, to have this all encompassing result. The form of such topological mass terms has been considered by Coleman \& Hill [16] in $\mathrm{QED}_{3}$ and by Reuter \& Dittrich [17], and Delbourgo \& Waites [15] in arbitrary dimensional space-time. It was shown by Coleman \& Hill in $\mathrm{QED}_{3}$, and in arbitrary dimensions by Delbourgo \& Waites, that the particular parity violating term considered is indeed the only possible form allowed for reasons of gauge invariance. This results in the strict correspondence between the number of photons in the interaction and the dimensionality of space-time.

Returning to a more general discussion we can observe that the interaction Lagrangian is very similar to the expression obtained in the scalar case, with the only significant discrepancies being the usual spinor factor $2^{[D / 2]}$, the different field invariant coefficients, and the possibility of parity violating terms. The introduction of the Bernoulli polynomial coefficients to describe the coefficients of the field invariants has exhibited the following correspondance:

$$
\begin{aligned}
B_{2 m}(0) & \text { for } \text { spin-0 propagator particles, } \\
2^{2 m} B_{2 m}\left(\frac{1}{2}\right) & \text { for }
\end{aligned}
$$

It is perhaps a rather large leap of faith to suggest that the Bernoulli polynomial parameter is related to the spin of the virtual propagator particles, but at least this does introduce a very compact and illuminating representation.

As a particular example we can consider the case of four photon scattering $(N=2)$ in arbitrary dimensional space-time. In this case the constraint over the $\left\{n_{i}\right\}$ implies that the only two terms allowed will have $n_{1}=2$ and $n_{2}=1$. Thus we immediately recover the standard electromagnetic field invariants

$$
\left(\operatorname{Tr} F^{2}\right)^{2}, \quad \text { and } \quad \operatorname{Tr} F^{4} \text {. }
$$

The coefficients can be evaluated easily from the general expression, and in the case of four photon scattering we obtain

$$
\mathcal{L}_{I}^{(1 / 2)}=\frac{1}{720} \frac{2^{[D / 2]}}{\left(m^{2}\right)^{4-D / 2}} \frac{e^{4}}{(4 \pi)^{D / 2}} \Gamma(4-D / 2)\left[14 \operatorname{Tr} F^{4}-5\left(\operatorname{Tr} F^{2}\right)^{2}\right] .
$$

Using a technique due to Ackhiezer \& Berestetskii [18 this result was verified from first principles by evaluating part of the Feynman amplitude for four photon scattering in arbitrary dimensional space-time. If we take the four dimensional case we obtain

$$
\mathcal{L}_{I}^{(1 / 2)}(D=4)=\frac{e^{4}}{2880 \pi^{2} m^{4}}\left[14 \operatorname{Tr} F^{4}-5\left(\operatorname{Tr} F^{2}\right)^{2}\right]
$$

which is the standard result obtained by Karplus \& Neuman [5] and Schwinger [9].

This concludes the discussion of $2 N$-photon scattering and to summarise we have obtained expressions (Eq. 6, 8,9) for the effective Lagrangian of the electromagnetic field which can describe photon-photon scattering to any order in arbitrary dimensions, where the virtual propagator particles may be either spin-0 or spin- $1 / 2$ particles. 


\section{$3 \quad 2 \mathrm{~N}+1-\mathrm{Photon}$ Case}

The case of interactions between an odd number of photons, although not perhaps describing pure scattering, is worthy of comment to complete the discussion. If we consider the case of an odd number of photons interacting via a virtual fermion loop then the case of even $D$ is trivial and results in a vanishing interaction Lagrangian as a consequence of Furry's Theorem.

For odd dimensions the situation is not quite so simple as Furry's theorem no longer applies when $D=4 k+1$ and there exists the possibility of having parity violating invariants similar in form to those which emerged in $2 \mathrm{~N}$-photon interactions. Delbourgo \& Waites [15] have shown that these terms are given by

$$
\frac{e^{2 N+1}}{2(2 N+1) !(4 \pi)^{2 N}} \epsilon_{\mu_{1} \cdots \mu_{D}} A^{\mu_{1}} F^{\mu_{2} \mu_{3}} \cdots F^{\mu_{D-1} \mu_{D}},
$$

for a $2 N+1$-photon process ( $N$ integral). For a given $2 N+1$-photon interaction this term is only present in a space-time with dimension $D$ given by

$$
D=2(2 N)+1
$$

which is the set of dimensionalities for which the charge conjugation operator does not exist. Consequently, we can write the effective electromagnetic field Lagrangian in this case as

$$
\mathcal{L}=-\frac{1}{4} \operatorname{Tr} F^{2}+\frac{e^{2 N+1}}{2(2 N+1) !(4 \pi)^{2 N}} \epsilon_{\mu_{1} \cdots \mu_{D}} A^{\mu_{1}} F^{\mu_{2} \mu_{3}} \cdots F^{\mu_{D-1} \mu_{D}},
$$

with the term of primary interest corresponding to a 3 photon interaction in $5 D$ when

$$
\mathcal{L}=-\frac{1}{4} \operatorname{Tr} F^{2}+\frac{e^{3}}{192 \pi^{2}} \epsilon_{\mu \nu \rho \sigma \lambda} A^{\mu} F^{\nu \rho} F^{\sigma \lambda} .
$$

As mentioned earlier Coleman \& Hill [16], and Delbourgo \& Waites [15], have shown that these are the only possible terms which may arise, due to gauge invariance considerations, and thus interactions of an odd number of photons in odd dimensional space-times only have a non-zero effective interaction Lagrangian in the specific cases described above.

\section{Relations Between Invariants}

When considering effective Lagrangians in arbitrary space-time dimensions it turns out that the electromagnetic field invariants can suffer some linear dependence in certain dimensionalities. This property is most easily illustrated in terms of a particular representation for the characteristic identities of the electromagnetic field tensor. This representation involves writing the characteristic equation in terms of polyno-

mials over traces of matrix powers. A Maple routine has previously been developed 
to obtain these results for any given dimension [19]. Some examples are listed below.

$$
\begin{array}{ccc}
D & =2 & F^{2}-\frac{1}{2} \mathrm{~T}_{2}=0 \\
D & =3 & F^{3}-\frac{1}{2} \mathrm{~T}_{2} F=0 \\
D & =4 & F^{4}-\frac{1}{2} \mathrm{~T}_{2} F^{2}+\left(\frac{1}{8} \mathrm{~T}_{2}^{2}-\frac{1}{4} \mathrm{~T}_{4}\right)=0 \\
D=5 & F^{5}-\frac{1}{2} \mathrm{~T}_{2} F^{3}+\left(\frac{1}{8} \mathrm{~T}_{2}^{2}-\frac{1}{4} \mathrm{~T}_{4}\right) F=0 \\
D=6 & F^{6}-\frac{1}{2} \mathrm{~T}_{2} F^{4}+\left(\frac{1}{8} \mathrm{~T}_{2}^{2}-\frac{1}{4} \mathrm{~T}_{4}\right) F^{2}-\frac{1}{48} \mathrm{~T}_{2}^{3}+\frac{1}{8} \mathrm{~T}_{2} \mathrm{~T}_{4}-\frac{1}{6} \mathrm{~T}_{6}=0,
\end{array}
$$

where $\mathrm{T}_{\mathrm{i}}=\operatorname{Tr}\left(F^{i}\right)$.

If we take as an example a four photon scattering interaction, the two electromagentic field invariants, $\operatorname{Tr} F^{4}$ and $\left(\operatorname{Tr} F^{2}\right)^{2}$, which are independent in $D=4$, are linearly dependent in $D=2,3$. As can be seen from the characteristic identities above, in these cases

$$
\left(\operatorname{Tr} F^{2}\right)^{2}=2 \operatorname{Tr} F^{4}
$$

Consequently the four-photon effective interaction Lagrangian simplifies in the case of $D=2$ to

$$
\mathcal{L}_{I}=\frac{e^{4}}{360 \pi m^{6}}\left(\operatorname{Tr} F^{2}\right)^{2}
$$

and in the case of $D=3$ to

$$
\mathcal{L}_{I}=\frac{e^{4}}{1920 \pi m^{5}}\left(\operatorname{Tr} F^{2}\right)^{2} .
$$

Similarly higher dimensional cases can have any linear dependence in the invariants removed by using the relevant characteristic identity, the general condition for linear dependence being simply that the number of photons in the interactions is greater than the space-time dimension. This is precisely the situation in which we would expect some linear depenence of the photon momenta due to the limited number of degrees of freedom. Thus it appears that the correspondence between $F^{\mu \nu}$ and the photon momenta $k^{\mu}$, via $F^{\mu \nu}=i\left(k^{\mu} A^{\nu}(k)-k^{\nu} A^{\mu}(k)\right)$, causes any linear dependence in the photon momenta to manifest itself as a linear dependence in the field invariants.

It is clear that at least in the simpler cases this linear dependence can be used to simplify the form of the effective Lagrangian. It would be interesting to know if the corresponding linear dependence in the photon momenta could be used to simplify calculation of the corresponding Feynman amplitude.

\section{Conclusions}

The discussion in this paper has focussed on low-energy photon interactions mediated by scalar and spinor propagators. We may also consider the possibility of interactions mediated by other forms of propagator particles. Photon-photon scattering mediated 
by electroweak propagators such as $W^{+}$and $W^{-}$particles has been considered by Okhlopkova [20], and the gauge invariance of the amplitude in the electroweak theory has been established by Boudjema [21]. However, there has apparently been no discussion of the invariant form of the Lagrangian in the literature. We noted earlier a possible correspondence in the Lagrangians between the Bernoulli polynomial parameter and the spin of the propagator particles. This relationship could be further tested by considering the form of the effective interaction Lagrangian for a photon scattering process mediated by a vector boson loop, i.e. $W^{ \pm}$spin- 1 particles. Taking the correspondence literally we might expect the effective Lagrangian to have a similar form to the expressions obtained for the scalar and spinor cases, except that the invariants would have coefficients involving, in some manner, the constants

$$
B_{2 m}(1)
$$

At this stage, this is obviously only pure supposition but continuing in the same vein we may be able to consider a slightly more believable generalisation. The effective Lagrangians obtained by Brown \& Duff [8] for the scalar and spinor cases indicated that essentially the only difference in the spinor case was the addition of an extra trace over spinor indices. Consequently we might predict that in the vector case similar expressions may result with the trace over spinor indices replaced by a trace of the form

$$
\operatorname{Tr} \exp [e s \Sigma \cdot F]
$$

where $\Sigma_{\mu}$ are the matrices equivalent to the Dirac matrices but for spin-1 particles. Realistically there may be Fadeev-Popov ghost contributions to take into account here, but the form of the 1-loop Lagrangians is certainly suggestive of the effective Lagrangian for photon scattering mediated by any electroweak propagator particle having a similar form to the expressions obtained in this paper.

\section{Acknowledgements}

After completion of the original preprint the authors were made aware, for the first time, of a paper by M.G. Schmidt and C. Schubert published in Phys. Lett. B 318:438 (1993) in which the generating functional for the effective Lagrangian (Eq. 9) was obtained. This result, obtained using a very different technique, apparently coincides with ours, and thus allows us some more confidence in our calculations. We note further that the paper of Schmidt and Schubert does not explicitly consider the form of the Lagrangian, nor did they consider the case of a scalar field. The authors are also grateful to C. Schubert for pointing out an error in the original preprint.

Finally, one of the authors, A.R., would like to acknowledge the generous support of a summer research studentship from the Department of Physics at the University of Tasmania. 


\section{References}

[1] O. Halpern, Phys. Rev. 44, 855 (1934).

[2] H. Euler and B. Kockel, Naturwiss. 23, 246 (1935).

[3] H. Euler, Ann. d. Physik 26, 398 (1936).

[4] W. Heisenberg and H. Euler, Zeits. f. Physik 98, 714 (1936).

[5] R. Karplus and M. Neuman, Phys. Rev. 80, 380 (1950).

[6] R. Karplus and M. Neuman, Phys. Rev. 83, 776 (1951).

[7] V. Berestetskii, E. Lifshitz, and L. Pitaevskii, Quantum Electrodynamics, Pergamon Press Ltd., 1982.

[8] M. Brown and M. Duff, Phys. Rev. D 11, 2124 (1975).

[9] J. Schwinger, Phys. Rev. 82, 664 (1951).

[10] J. Schwinger, Particles, Sources, and Fields, Vol. 2, Addison-Wesley, 1973.

[11] R. Delbourgo and T. Matsuki, J. Math. Phys. 26, 1334 (1985).

[12] L. Alvarez-Gaume and E. Witten, Nucl. Phys. B 234, 269 (1983).

[13] R. Endo and M. Takao, Prog. Theor. Phys. 73, 803 (1985).

[14] A. Prudnikov, Y. Brychkov, and O. Marichev, Integrals and Series, Vol. 3, Gordon \& Breach Science Publishers, 1990.

[15] R. Delbourgo and A. Waites, Aust. J. Phys. 47, 465 (1994).

[16] S. Coleman and B. Hill, Phys. Lett. B 159, 184 (1985).

[17] M. Reuter and W. Dittrich, Phys. Rev. D 33, 601 (1975).

[18] A. Ackhiezer and V. Berestetskii, Quantum Electrodynamics, Interscience, 1965.

[19] R. Delbourgo, preprint: UTAS-PHYS-93-42 (1993).

[20] V. Okhlopkova, Nucl. Phys. B 108, 170 (1976).

[21] F. Boudjema, Phys. Lett. B 187, 362 (1975). 